Acta Crystallographica Section E

Structure Reports

Online

ISSN 1600-5368

\section{3-Methyl-1-(3-nitrophenyl)-5-phenyl-4,5- dihydro-1H-pyrazole}

\section{Jun-qiang Chen, ${ }^{\mathrm{a} *}$ He-ping $\mathrm{Li}^{\mathrm{b}}{ }^{\mathrm{b}}$ Chang-shan Huang ${ }^{\mathrm{a}}$ and Jin-ying $\mathbf{W u}^{\mathrm{a}}$}

${ }^{a}$ Energy Research Institute Co Ltd, Henan Academy of Sciences, Zhengzhou 450000, People's Republic of China, and 'bchool of Chemistry and Biological Engineering, Guilin University of Technology, People's Republic of China

Correspondence e-mail: junqiangchen2009@126.com

Received 6 August 2009; accepted 9 August 2009

Key indicators: single-crystal X-ray study; $T=296 \mathrm{~K}$; mean $\sigma(\mathrm{C}-\mathrm{C})=0.003 \AA$; $R$ factor $=0.047 ; w R$ factor $=0.128 ;$ data-to-parameter ratio $=15.9$.

In the title compound, $\mathrm{C}_{16} \mathrm{H}_{15} \mathrm{~N}_{3} \mathrm{O}_{2}$, the planar [maximum deviation 0.156 (2) $\AA$ ] pyrazoline ring is nearly coplanar with the 3-nitrophenyl group and is approximately perpendicular to the phenyl ring, making dihedral angles of $3.80(8)$ and $80.58(10)^{\circ}$, respectively. Weak intermolecular $\mathrm{C}-\mathrm{H} \cdots \mathrm{O}$ hydrogen bonding is present in the crystal structure.

\section{Related literature}

For applications of pyrazoline derivatives, see: Hatheway et al. (1978); Mahajan et al. (1991); Sobczak \& Pawlaczyk (1998).

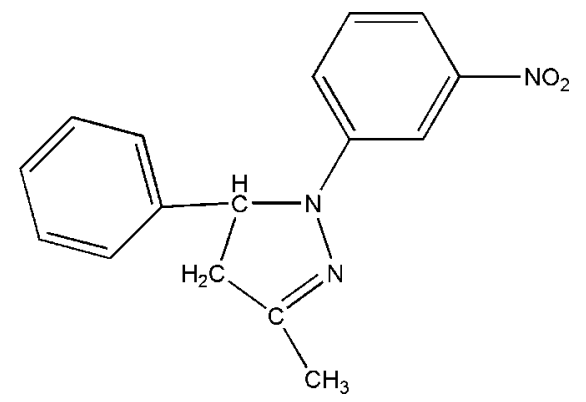

Monoclinic, $P 2_{1} / n$

$a=12.0173(4) \AA$

$b=7.9324(2) \AA$

$c=15.4944(5) \AA$

$\beta=99.160$ (2)

$V=1458.18(8) \AA^{3}$

Data collection

Bruker SMART CCD area-detector diffractometer

Absorption correction: none

10272 measured reflections

Refinement

$R\left[F^{2}>2 \sigma\left(F^{2}\right)\right]=0.047$

$w R\left(F^{2}\right)=0.128$

$S=1.00$

3014 reflections

$Z=4$

Mo $K \alpha$ radiation

$\mu=0.09 \mathrm{~mm}^{-1}$

$T=296 \mathrm{~K}$

$0.36 \times 0.18 \times 0.07 \mathrm{~mm}$ 1648 reflections with $I>2 \sigma(I)$

$R_{\text {int }}=0.034$

90 parameters

$\mathrm{H}$-atom parameters constrained

$\Delta \rho_{\max }=0.14 \mathrm{e} \AA^{-3}$

$\Delta \rho_{\min }=-0.20 \mathrm{e} \AA^{-3}$

Table 1

Hydrogen-bond geometry $\left(\AA{ }^{\circ}\right)$.

\begin{tabular}{lllll}
\hline$D-\mathrm{H} \cdots A$ & $D-\mathrm{H}$ & $\mathrm{H} \cdots A$ & $D \cdots A$ & $D-\mathrm{H} \cdots A$ \\
\hline $\mathrm{C} 14-\mathrm{H} 14 A \cdots \mathrm{O} 1^{\mathrm{i}}$ & 0.93 & 2.51 & $3.245(2)$ & 136 \\
\hline
\end{tabular}

Symmetry code: (i) $-x+\frac{3}{2}, y+\frac{1}{2},-z+\frac{1}{2}$.

Data collection: SMART (Bruker, 1998); cell refinement: SAINT (Bruker, 1998); data reduction: SAINT (Bruker, 1998); program(s) used to solve structure: SHELXS97 (Sheldrick, 2008); program(s) used to refine structure: SHELXL97 (Sheldrick, 2008); molecular graphics: ORTEP-3 for Windows (Farrugia, 1997); software used to prepare material for publication: WinGX (Farrugia, 1999).

Supplementary data and figures for this paper are available from the IUCr electronic archives (Reference: XU2579).

\title{
References
}

Bruker (1998). SMART and SAINT. Bruker AXS Inc., Madison, Wisconsin, USA.

Farrugia, L. J. (1997). J. Appl. Cryst. 30, 565.

Farrugia, L. J. (1999). J. Appl. Cryst. 32, 837-838.

Hatheway, G. J., Hansch, C., Kim, K. H., Milstein, S. R., Schmidt, C. L., Smith, R. N. \& Quinn, F. R. (1978). J. Med. Chem. 21, 563-567.

Mahajan, R. N., Havaldar, F. H. \& Fernandes, P. S. (1991). J. Indian Chem. Soc. 68, 245-246.

Sheldrick, G. M. (2008). Acta Cryst. A64, 112-122.

Sobczak, H. \& Pawlaczyk, J. (1998). Acta Pol. Pharm. 55, 279-283.

\section{Experimental}

Crystal data

$\mathrm{C}_{16} \mathrm{H}_{15} \mathrm{~N}_{3} \mathrm{O}_{2} \quad M_{r}=281.31$ 


\section{supporting information}

Acta Cryst. (2009). E65, o2156 [doi:10.1107/S1600536809031390]

\section{3-Methyl-1-(3-nitrophenyl)-5-phenyl-4,5-dihydro-1 H-pyrazole \\ Jun-qiang Chen, He-ping Li, Chang-shan Huang and Jin-ying Wu}

\section{S1. Comment}

The derivatives of pyrazoline are mostly used in medicine, for example as antitumor (Hatheway et al., 1978), analgesic (Sobczak \& Pawlaczyk, 1998), and antimicrobial (Mahajan et al., 1991) agents. As part of our work, the title compound is recently synthesized in our group and its crystal structure is reported here.

The pyrazoline ring and the 3-nitrophenyl ring are nearly coplanar, making a dihedral angle of $3.80(8)^{\circ}$, while the dihedral angle between the pyrazoline ring and the $\mathrm{C} 1$-phenyl ring is $80.58(10)^{\circ}$ (Fig. 1). Intermolecular weak $\mathrm{C}-\mathrm{H} \cdots \mathrm{O}$ hydrogen bonding is present in the crystal structure (Fig. 2 and Table 1).

\section{S2. Experimental}

3-Nitrophenylhydrazine ( $1 \mathrm{mmol}, 0.153 \mathrm{~g})$ was dissolved in anhydrous ethanol $(15 \mathrm{ml})$. The mixture was stirred for several $\mathrm{min}$ at $351 \mathrm{~K}$, benzylideneacetone $(1 \mathrm{mmol}, 0.146 \mathrm{~g})$ in ethanol $(8 \mathrm{ml})$ was added dropwise and the mixture was stirred at refluxing temperature for $2 \mathrm{~h}$. The product was isolated and recrystallized from methanol, red single crystals were obtained after $2 \mathrm{~d}$.

\section{S3. Refinement}

All $\mathrm{H}$ atoms were positioned geometrically and refined as riding with $\mathrm{C}-\mathrm{H}=0.93$ (aromatic), 0.97 (methylene), 0.98 (methine) and $0.96 \AA$ (methyl), with $U_{\text {iso }}(\mathrm{H})=1.5 U_{\mathrm{eq}}(\mathrm{C})$ for methyl $\mathrm{H}$ atoms and $1.2 U_{\mathrm{eq}}(\mathrm{C})$ for the others. 


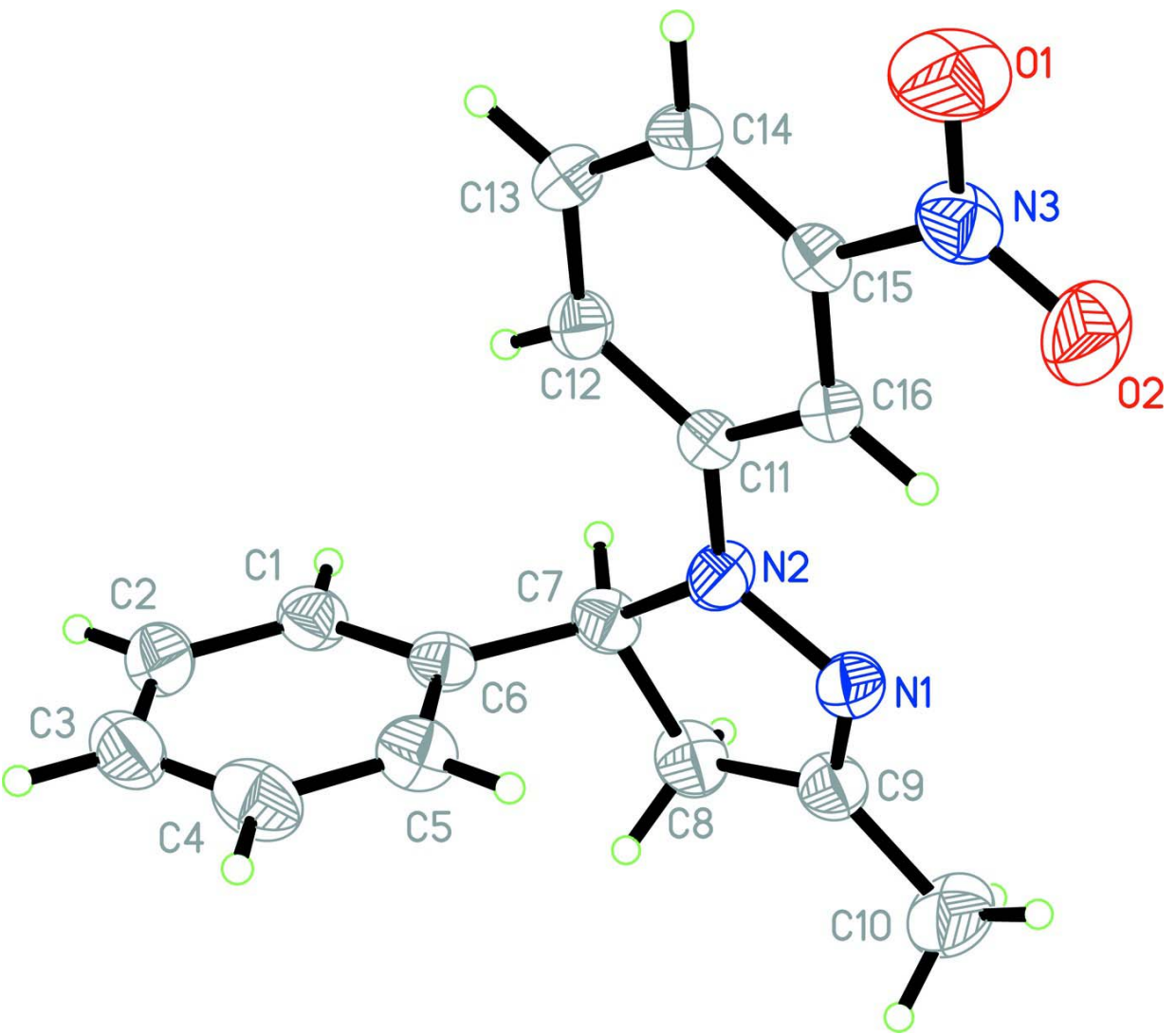

\section{Figure 1}

The molecular structure of the compound. The displacement ellipsoids are drawn at the $30 \%$ probability level. 


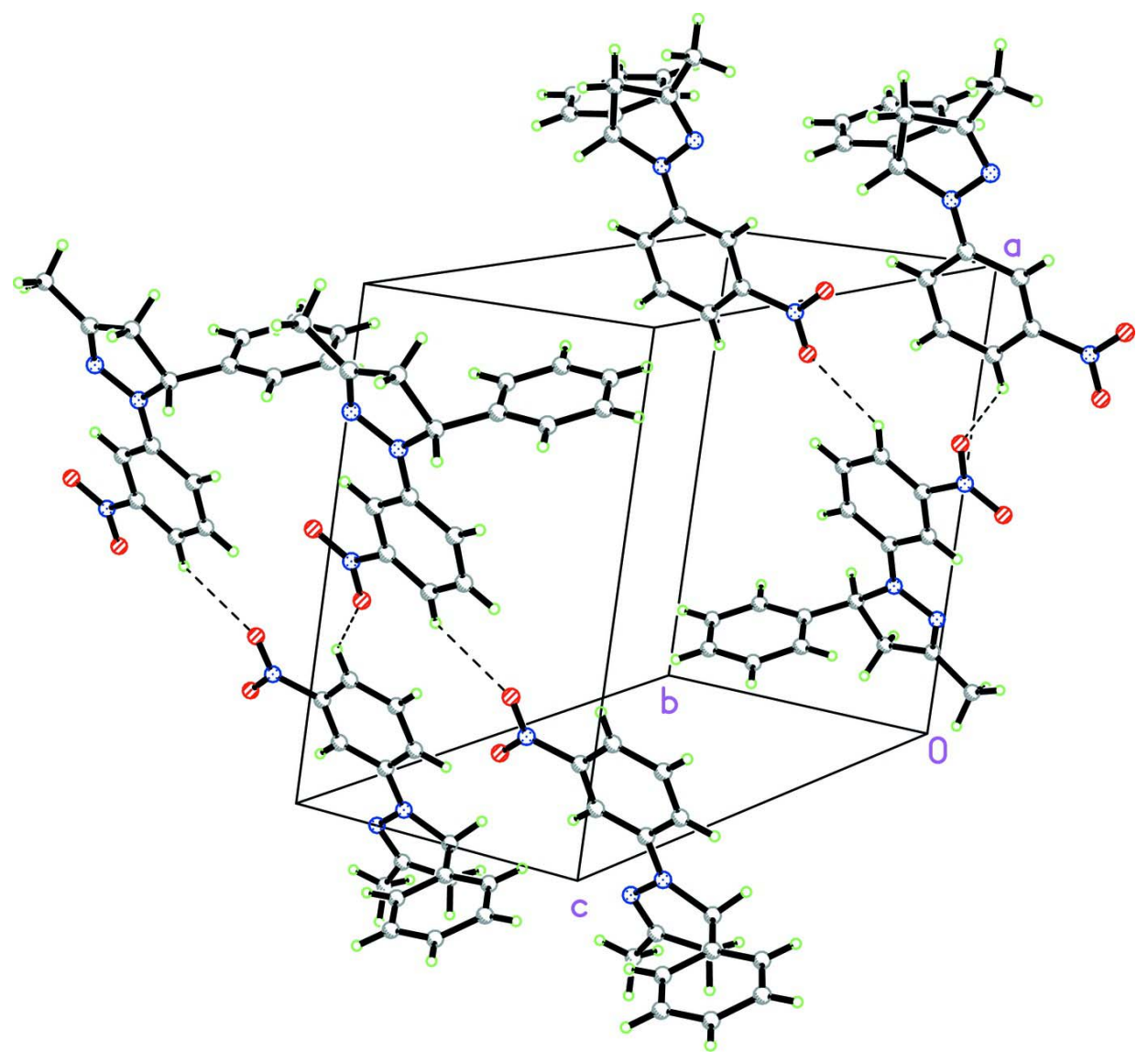

Figure 2

Packing of (I), showing the intermolecular hydrogen bonds as dashed lines.

\section{3-Methyl-1-(3-nitrophenyl)-5-phenyl-4,5-dihydro-1 $H$-pyrazole}

\section{Crystal data}

$\mathrm{C}_{16} \mathrm{H}_{15} \mathrm{~N}_{3} \mathrm{O}_{2}$

$M_{r}=281.31$

Monoclinic, $P 2{ }_{1} / n$

Hall symbol: -P 2 yn

$a=12.0173(4) \AA$

$b=7.9324(2) \AA$

$c=15.4944(5) \AA$

$\beta=99.160(2)^{\circ}$

$V=1458.18(8) \AA^{3}$

$Z=4$

\section{Data collection}

Bruker SMART CCD area-detector diffractometer

Radiation source: fine-focus sealed tube

Graphite monochromator

$\omega$ scans

10272 measured reflections

3014 independent reflections
$F(000)=592$

$D_{\mathrm{x}}=1.281 \mathrm{Mg} \mathrm{m}^{-3}$

Mo $K \alpha$ radiation, $\lambda=0.71073 \AA$

Cell parameters from 1824 reflections

$\theta=2.6-26.5^{\circ}$

$\mu=0.09 \mathrm{~mm}^{-1}$

$T=296 \mathrm{~K}$

Plate, red

$0.36 \times 0.18 \times 0.07 \mathrm{~mm}$

1648 reflections with $I>2 \sigma(I)$

$R_{\text {int }}=0.034$

$\theta_{\text {max }}=26.5^{\circ}, \theta_{\text {min }}=2.0^{\circ}$

$h=-14 \rightarrow 15$

$k=-9 \rightarrow 8$

$l=-18 \rightarrow 19$ 


\section{Refinement}

Refinement on $F^{2}$

Least-squares matrix: full

$R\left[F^{2}>2 \sigma\left(F^{2}\right)\right]=0.047$

$w R\left(F^{2}\right)=0.128$

$S=1.00$

3014 reflections

190 parameters

0 restraints

Primary atom site location: structure-invariant direct methods
Secondary atom site location: difference Fourier map

Hydrogen site location: inferred from neighbouring sites

$\mathrm{H}$-atom parameters constrained

$w=1 /\left[\sigma^{2}\left(F_{\mathrm{o}}^{2}\right)+(0.0621 P)^{2}\right]$ where $P=\left(F_{\mathrm{o}}^{2}+2 F_{\mathrm{c}}^{2}\right) / 3$

$(\Delta / \sigma)_{\max }<0.001$

$\Delta \rho_{\max }=0.14 \mathrm{e} \AA^{-3}$

$\Delta \rho_{\min }=-0.20$ e $\AA^{-3}$

Special details

Geometry. All e.s.d.'s (except the e.s.d. in the dihedral angle between two l.s. planes) are estimated using the full covariance matrix. The cell e.s.d.'s are taken into account individually in the estimation of e.s.d.'s in distances, angles and torsion angles; correlations between e.s.d.'s in cell parameters are only used when they are defined by crystal symmetry. An approximate (isotropic) treatment of cell e.s.d.'s is used for estimating e.s.d.'s involving 1.s. planes.

Refinement. Refinement of $F^{2}$ against ALL reflections. The weighted $R$-factor $w R$ and goodness of fit $S$ are based on $F^{2}$, conventional $R$-factors $R$ are based on $F$, with $F$ set to zero for negative $F^{2}$. The threshold expression of $F^{2}>\sigma\left(F^{2}\right)$ is used only for calculating $R$-factors (gt) etc. and is not relevant to the choice of reflections for refinement. $R$-factors based on $F^{2}$ are statistically about twice as large as those based on $F$, and $R$ - factors based on ALL data will be even larger.

Fractional atomic coordinates and isotropic or equivalent isotropic displacement parameters $\left(\AA^{2}\right)$

\begin{tabular}{|c|c|c|c|c|}
\hline & $x$ & $y$ & $z$ & $U_{\text {iso }} * / U_{\text {eq }}$ \\
\hline $\mathrm{C} 11$ & $0.40009(13)$ & 0.17187 (19) & $0.06087(10)$ & $0.0433(4)$ \\
\hline N2 & $0.29773(12)$ & $0.18897(16)$ & $0.00749(10)$ & $0.0578(4)$ \\
\hline $\mathrm{C} 15$ & $0.54447(14)$ & 0.00161 (19) & $0.13960(10)$ & $0.0454(4)$ \\
\hline $\mathrm{C} 7$ & $0.24060(14)$ & $0.3481(2)$ & $-0.02008(11)$ & $0.0535(5)$ \\
\hline H7A & 0.2879 & 0.4156 & -0.0529 & $0.064 *$ \\
\hline C16 & $0.44095(13)$ & 0.01314 (19) & $0.08743(10)$ & $0.0425(4)$ \\
\hline H16A & 0.3991 & -0.0832 & 0.0703 & $0.051 *$ \\
\hline N1 & $0.23515(12)$ & $0.04742(18)$ & $-0.02230(9)$ & $0.0548(4)$ \\
\hline $\mathrm{C} 12$ & $0.46581(14)$ & $0.3125(2)$ & $0.08824(11)$ & $0.0519(5)$ \\
\hline $\mathrm{H} 4 \mathrm{~A}$ & 0.4395 & 0.4197 & 0.0714 & $0.062 *$ \\
\hline C6 & $0.21138(13)$ & $0.4493(2)$ & $0.05545(11)$ & $0.0472(4)$ \\
\hline C13 & $0.56934(14)$ & $0.2941(2)$ & $0.13993(12)$ & $0.0561(5)$ \\
\hline H13A & 0.6121 & 0.3895 & 0.1572 & $0.067 *$ \\
\hline N3 & $0.58488(14)$ & $-0.1672(2)$ & $0.16800(11)$ & $0.0619(4)$ \\
\hline $\mathrm{C} 1$ & $0.21969(14)$ & $0.6221(2)$ & $0.05691(13)$ & $0.0584(5)$ \\
\hline H1B & 0.2456 & 0.6777 & 0.0111 & $0.070^{*}$ \\
\hline $\mathrm{O} 1$ & $0.67543(13)$ & $-0.17917(18)$ & $0.21576(11)$ & $0.0990(6)$ \\
\hline $\mathrm{C} 14$ & $0.61085(14)$ & $0.1383(2)$ & $0.16652(11)$ & $0.0536(5)$ \\
\hline H14A & 0.6809 & 0.1259 & 0.2013 & $0.064 *$ \\
\hline $\mathrm{O} 2$ & $0.52813(13)$ & $-0.28871(17)$ & $0.14279(11)$ & $0.0905(5)$ \\
\hline $\mathrm{C} 5$ & $0.17261(15)$ & $0.3710(3)$ & $0.12393(13)$ & $0.0650(5)$ \\
\hline H5A & 0.1664 & 0.2542 & 0.1241 & $0.078 *$ \\
\hline $\mathrm{C} 8$ & $0.13676(16)$ & $0.2830(2)$ & $-0.08217(13)$ & $0.0684(6)$ \\
\hline $\mathrm{H} 8 \mathrm{~A}$ & 0.0675 & 0.3240 & -0.0649 & $0.082 *$ \\
\hline H8B & 0.1391 & 0.3168 & -0.1420 & $0.082 *$ \\
\hline
\end{tabular}


supporting information

$\begin{array}{lllll}\text { C9 } & 0.14648(15) & 0.0972(2) & -0.07219(12) & 0.0591(5) \\ \text { C4 } & 0.14288(17) & 0.4619(3) & 0.19199(14) & 0.0777(6) \\ \text { H12A } & 0.1166 & 0.4065 & 0.2377 & 0.093^{*} \\ \text { C2 } & 0.19016(16) & 0.7141(3) & 0.12524(16) & 0.0729(6) \\ \text { H2A } & 0.1963 & 0.8310 & 0.1255 & 0.088^{*} \\ \text { C3 } & 0.15175(17) & 0.6329(4) & 0.19279(14) & 0.0777(6) \\ \text { H3A } & 0.1318 & 0.6945 & 0.2391 & 0.093^{*} \\ \text { C10 } & 0.06227(18) & -0.0247(3) & -0.11675(16) & 0.0957(8) \\ \text { H10A } & 0.0856 & -0.1375 & -0.1002 & 0.144^{*} \\ \text { H10B } & 0.0567 & -0.0124 & -0.1789 & 0.144^{*} \\ \text { H10C } & -0.0098 & -0.0029 & -0.0999 & 0.144^{*}\end{array}$

Atomic displacement parameters $\left(\AA^{2}\right)$

\begin{tabular}{lllllll}
\hline & $U^{11}$ & $U^{22}$ & $U^{33}$ & $U^{12}$ & $U^{13}$ & $U^{23}$ \\
\hline C11 & $0.0398(10)$ & $0.0436(10)$ & $0.0470(10)$ & $0.0024(8)$ & $0.0079(8)$ & $-0.0019(8)$ \\
N2 & $0.0495(9)$ & $0.0418(8)$ & $0.0756(11)$ & $0.0045(7)$ & $-0.0098(8)$ & $-0.0003(7)$ \\
C15 & $0.0460(10)$ & $0.0452(10)$ & $0.0457(10)$ & $0.0054(8)$ & $0.0097(8)$ & $0.0021(8)$ \\
C7 & $0.0505(11)$ & $0.0526(11)$ & $0.0567(11)$ & $0.0095(9)$ & $0.0057(9)$ & $0.0079(9)$ \\
C16 & $0.0402(10)$ & $0.0422(10)$ & $0.0451(10)$ & $-0.0005(7)$ & $0.0066(8)$ & $-0.0016(7)$ \\
N1 & $0.0486(9)$ & $0.0553(9)$ & $0.0586(10)$ & $-0.0003(7)$ & $0.0028(8)$ & $-0.0044(7)$ \\
C12 & $0.0524(11)$ & $0.0414(10)$ & $0.0613(12)$ & $0.0019(8)$ & $0.0072(9)$ & $-0.0004(8)$ \\
C6 & $0.0393(10)$ & $0.0503(11)$ & $0.0509(11)$ & $0.0056(8)$ & $0.0044(8)$ & $0.0076(8)$ \\
C13 & $0.0483(11)$ & $0.0520(11)$ & $0.0669(13)$ & $-0.0111(9)$ & $0.0059(10)$ & $-0.0090(9)$ \\
N3 & $0.0575(10)$ & $0.0596(11)$ & $0.0673(11)$ & $0.0122(9)$ & $0.0054(9)$ & $0.0115(9)$ \\
C1 & $0.0515(11)$ & $0.0548(12)$ & $0.0698(13)$ & $0.0035(9)$ & $0.0127(10)$ & $0.0041(10)$ \\
O1 & $0.0680(10)$ & $0.0945(12)$ & $0.1213(13)$ & $0.0163(8)$ & $-0.0254(10)$ & $0.0320(9)$ \\
C14 & $0.0414(10)$ & $0.0600(12)$ & $0.0576(12)$ & $0.0024(9)$ & $0.0020(9)$ & $-0.0024(9)$ \\
O2 & $0.0924(11)$ & $0.0471(8)$ & $0.1237(14)$ & $0.0038(8)$ & $-0.0081(10)$ & $0.0076(8)$ \\
C5 & $0.0650(13)$ & $0.0650(12)$ & $0.0661(13)$ & $0.0024(10)$ & $0.0139(11)$ & $0.0113(11)$ \\
C8 & $0.0642(13)$ & $0.0779(14)$ & $0.0579(13)$ & $0.0157(11)$ & $-0.0064(10)$ & $-0.0013(10)$ \\
C9 & $0.0494(11)$ & $0.0694(13)$ & $0.0555(12)$ & $0.0051(10)$ & $-0.0012(10)$ & $-0.0062(10)$ \\
C4 & $0.0732(15)$ & $0.1004(19)$ & $0.0631(15)$ & $0.0073(13)$ & $0.0215(12)$ & $0.0091(13)$ \\
C2 & $0.0633(13)$ & $0.0636(13)$ & $0.0913(17)$ & $0.0054(11)$ & $0.0102(13)$ & $-0.0148(12)$ \\
C3 & $0.0609(13)$ & $0.1077(19)$ & $0.0644(15)$ & $0.0120(13)$ & $0.0094(11)$ & $-0.0195(14)$ \\
C10 & $0.0690(15)$ & $0.1000(18)$ & $0.1050(19)$ & $-0.0036(12)$ & $-0.0269(13)$ & $-0.0190(14)$ \\
& & & & & & \\
\hline
\end{tabular}

Geometric parameters $\left(\AA,{ }^{\circ}\right)$

\begin{tabular}{llll}
\hline $\mathrm{C} 11-\mathrm{N} 2$ & $1.375(2)$ & $\mathrm{N} 3-\mathrm{O} 2$ & $1.2093(18)$ \\
$\mathrm{C} 11-\mathrm{C} 16$ & $1.390(2)$ & $\mathrm{N} 3-\mathrm{O} 1$ & $1.2185(19)$ \\
$\mathrm{C} 11-\mathrm{C} 12$ & $1.393(2)$ & $\mathrm{C} 1-\mathrm{C} 2$ & $1.378(3)$ \\
$\mathrm{N} 2-\mathrm{N} 1$ & $1.3888(18)$ & $\mathrm{C} 1-\mathrm{H} 1 \mathrm{~B}$ & 0.9300 \\
$\mathrm{~N} 2-\mathrm{C} 7$ & $1.4678(19)$ & $\mathrm{C} 14-\mathrm{H} 14 \mathrm{~A}$ & 0.9300 \\
$\mathrm{C} 15-\mathrm{C} 14$ & $1.371(2)$ & $\mathrm{C} 5-\mathrm{C} 4$ & $1.371(3)$ \\
$\mathrm{C} 15-\mathrm{C} 16$ & $1.374(2)$ & $\mathrm{C} 5-\mathrm{H} 5 \mathrm{~A}$ & 0.9300 \\
$\mathrm{C} 15-\mathrm{N} 3$ & $1.468(2)$ & $\mathrm{C} 8-\mathrm{C} 9$ & $1.485(3)$ \\
$\mathrm{C} 7-\mathrm{C} 6$ & $1.506(2)$ & $\mathrm{C} 8-\mathrm{H} 8 \mathrm{~A}$ & 0.9700
\end{tabular}




\begin{tabular}{|c|c|c|c|}
\hline $\mathrm{C} 7-\mathrm{C} 8$ & $1.539(2)$ & $\mathrm{C} 8-\mathrm{H} 8 \mathrm{~B}$ & 0.9700 \\
\hline $\mathrm{C} 7-\mathrm{H} 7 \mathrm{~A}$ & 0.9800 & $\mathrm{C} 9-\mathrm{C} 10$ & $1.488(3)$ \\
\hline $\mathrm{C} 16-\mathrm{H} 16 \mathrm{~A}$ & 0.9300 & $\mathrm{C} 4-\mathrm{C} 3$ & $1.360(3)$ \\
\hline $\mathrm{N} 1-\mathrm{C} 9$ & $1.275(2)$ & $\mathrm{C} 4-\mathrm{H} 12 \mathrm{~A}$ & 0.9300 \\
\hline $\mathrm{C} 12-\mathrm{C} 13$ & $1.376(2)$ & $\mathrm{C} 2-\mathrm{C} 3$ & $1.370(3)$ \\
\hline $\mathrm{C} 12-\mathrm{H} 4 \mathrm{~A}$ & 0.9300 & $\mathrm{C} 2-\mathrm{H} 2 \mathrm{~A}$ & 0.9300 \\
\hline $\mathrm{C} 6-\mathrm{C} 5$ & $1.373(2)$ & $\mathrm{C} 3-\mathrm{H} 3 \mathrm{~A}$ & 0.9300 \\
\hline $\mathrm{C} 6-\mathrm{C} 1$ & $1.374(2)$ & $\mathrm{C} 10-\mathrm{H} 10 \mathrm{~A}$ & 0.9600 \\
\hline $\mathrm{C} 13-\mathrm{C} 14$ & $1.371(2)$ & $\mathrm{C} 10-\mathrm{H} 10 \mathrm{~B}$ & 0.9600 \\
\hline $\mathrm{C} 13-\mathrm{H} 13 \mathrm{~A}$ & 0.9300 & $\mathrm{C} 10-\mathrm{H} 10 \mathrm{C}$ & 0.9600 \\
\hline $\mathrm{N} 2-\mathrm{C} 11-\mathrm{C} 16$ & $120.49(14)$ & $\mathrm{C} 6-\mathrm{C} 1-\mathrm{H} 1 \mathrm{~B}$ & 119.5 \\
\hline $\mathrm{N} 2-\mathrm{C} 11-\mathrm{C} 12$ & $120.89(14)$ & $\mathrm{C} 2-\mathrm{C} 1-\mathrm{H} 1 \mathrm{~B}$ & 119.5 \\
\hline $\mathrm{C} 16-\mathrm{C} 11-\mathrm{C} 12$ & $118.61(15)$ & $\mathrm{C} 15-\mathrm{C} 14-\mathrm{C} 13$ & $117.08(16)$ \\
\hline $\mathrm{C} 11-\mathrm{N} 2-\mathrm{N} 1$ & $120.35(13)$ & $\mathrm{C} 15-\mathrm{C} 14-\mathrm{H} 14 \mathrm{~A}$ & 121.5 \\
\hline $\mathrm{C} 11-\mathrm{N} 2-\mathrm{C} 7$ & $126.29(14)$ & $\mathrm{C} 13-\mathrm{C} 14-\mathrm{H} 14 \mathrm{~A}$ & 121.5 \\
\hline $\mathrm{N} 1-\mathrm{N} 2-\mathrm{C} 7$ & $113.28(13)$ & $\mathrm{C} 4-\mathrm{C} 5-\mathrm{C} 6$ & $121.3(2)$ \\
\hline $\mathrm{C} 14-\mathrm{C} 15-\mathrm{C} 16$ & $123.67(15)$ & $\mathrm{C} 4-\mathrm{C} 5-\mathrm{H} 5 \mathrm{~A}$ & 119.4 \\
\hline $\mathrm{C} 14-\mathrm{C} 15-\mathrm{N} 3$ & $118.79(15)$ & $\mathrm{C} 6-\mathrm{C} 5-\mathrm{H} 5 \mathrm{~A}$ & 119.4 \\
\hline $\mathrm{C} 16-\mathrm{C} 15-\mathrm{N} 3$ & $117.54(15)$ & $\mathrm{C} 9-\mathrm{C} 8-\mathrm{C} 7$ & $103.05(14)$ \\
\hline $\mathrm{N} 2-\mathrm{C} 7-\mathrm{C} 6$ & $112.85(14)$ & $\mathrm{C} 9-\mathrm{C} 8-\mathrm{H} 8 \mathrm{~A}$ & 111.2 \\
\hline $\mathrm{N} 2-\mathrm{C} 7-\mathrm{C} 8$ & $100.85(13)$ & $\mathrm{C} 7-\mathrm{C} 8-\mathrm{H} 8 \mathrm{~A}$ & 111.2 \\
\hline $\mathrm{C} 6-\mathrm{C} 7-\mathrm{C} 8$ & $113.49(13)$ & $\mathrm{C} 9-\mathrm{C} 8-\mathrm{H} 8 \mathrm{~B}$ & 111.2 \\
\hline $\mathrm{N} 2-\mathrm{C} 7-\mathrm{H} 7 \mathrm{~A}$ & 109.8 & $\mathrm{C} 7-\mathrm{C} 8-\mathrm{H} 8 \mathrm{~B}$ & 111.2 \\
\hline $\mathrm{C} 6-\mathrm{C} 7-\mathrm{H} 7 \mathrm{~A}$ & 109.8 & $\mathrm{H} 8 \mathrm{~A}-\mathrm{C} 8-\mathrm{H} 8 \mathrm{~B}$ & 109.1 \\
\hline $\mathrm{C} 8-\mathrm{C} 7-\mathrm{H} 7 \mathrm{~A}$ & 109.8 & $\mathrm{~N} 1-\mathrm{C} 9-\mathrm{C} 8$ & $114.49(16)$ \\
\hline $\mathrm{C} 15-\mathrm{C} 16-\mathrm{C} 11$ & $118.59(15)$ & $\mathrm{N} 1-\mathrm{C} 9-\mathrm{C} 10$ & $121.42(18)$ \\
\hline $\mathrm{C} 15-\mathrm{C} 16-\mathrm{H} 16 \mathrm{~A}$ & 120.7 & $\mathrm{C} 8-\mathrm{C} 9-\mathrm{C} 10$ & $124.09(17)$ \\
\hline $\mathrm{C} 11-\mathrm{C} 16-\mathrm{H} 16 \mathrm{~A}$ & 120.7 & $\mathrm{C} 3-\mathrm{C} 4-\mathrm{C} 5$ & $120.1(2)$ \\
\hline $\mathrm{C} 9-\mathrm{N} 1-\mathrm{N} 2$ & $107.90(15)$ & $\mathrm{C} 3-\mathrm{C} 4-\mathrm{H} 12 \mathrm{~A}$ & 120.0 \\
\hline $\mathrm{C} 13-\mathrm{C} 12-\mathrm{C} 11$ & $120.57(15)$ & $\mathrm{C} 5-\mathrm{C} 4-\mathrm{H} 12 \mathrm{~A}$ & 120.0 \\
\hline $\mathrm{C} 13-\mathrm{C} 12-\mathrm{H} 4 \mathrm{~A}$ & 119.7 & $\mathrm{C} 3-\mathrm{C} 2-\mathrm{C} 1$ & $119.8(2)$ \\
\hline $\mathrm{C} 11-\mathrm{C} 12-\mathrm{H} 4 \mathrm{~A}$ & 119.7 & $\mathrm{C} 3-\mathrm{C} 2-\mathrm{H} 2 \mathrm{~A}$ & 120.1 \\
\hline $\mathrm{C} 5-\mathrm{C} 6-\mathrm{C} 1$ & $118.09(17)$ & $\mathrm{C} 1-\mathrm{C} 2-\mathrm{H} 2 \mathrm{~A}$ & 120.1 \\
\hline $\mathrm{C} 5-\mathrm{C} 6-\mathrm{C} 7$ & $120.62(16)$ & $\mathrm{C} 4-\mathrm{C} 3-\mathrm{C} 2$ & $119.8(2)$ \\
\hline $\mathrm{C} 1-\mathrm{C} 6-\mathrm{C} 7$ & $121.27(16)$ & $\mathrm{C} 4-\mathrm{C} 3-\mathrm{H} 3 \mathrm{~A}$ & 120.1 \\
\hline $\mathrm{C} 14-\mathrm{C} 13-\mathrm{C} 12$ & $121.49(16)$ & $\mathrm{C} 2-\mathrm{C} 3-\mathrm{H} 3 \mathrm{~A}$ & 120.1 \\
\hline $\mathrm{C} 14-\mathrm{C} 13-\mathrm{H} 13 \mathrm{~A}$ & 119.3 & $\mathrm{C} 9-\mathrm{C} 10-\mathrm{H} 10 \mathrm{~A}$ & 109.5 \\
\hline $\mathrm{C} 12-\mathrm{C} 13-\mathrm{H} 13 \mathrm{~A}$ & 119.3 & $\mathrm{C} 9-\mathrm{C} 10-\mathrm{H} 10 \mathrm{~B}$ & 109.5 \\
\hline $\mathrm{O} 2-\mathrm{N} 3-\mathrm{O} 1$ & $122.49(16)$ & $\mathrm{H} 10 \mathrm{~A}-\mathrm{C} 10-\mathrm{H} 10 \mathrm{~B}$ & 109.5 \\
\hline $\mathrm{O} 2-\mathrm{N} 3-\mathrm{C} 15$ & $119.20(15)$ & $\mathrm{C} 9-\mathrm{C} 10-\mathrm{H} 10 \mathrm{C}$ & 109.5 \\
\hline $\mathrm{O} 1-\mathrm{N} 3-\mathrm{C} 15$ & $118.31(16)$ & $\mathrm{H} 10 \mathrm{~A}-\mathrm{C} 10-\mathrm{H} 10 \mathrm{C}$ & 109.5 \\
\hline $\mathrm{C} 6-\mathrm{C} 1-\mathrm{C} 2$ & $120.93(19)$ & $\mathrm{H} 10 \mathrm{~B}-\mathrm{C} 10-\mathrm{H} 10 \mathrm{C}$ & 109.5 \\
\hline
\end{tabular}

Hydrogen-bond geometry $\left(\AA,{ }^{\circ}\right)$

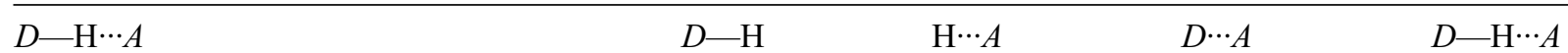


Symmetry code: (i) $-x+3 / 2, y+1 / 2,-z+1 / 2$. 